\title{
A NOTE ON FIRST PASSAGE PROBABILITIES OF A LÉVY PROCESS REFLECTED AT A GENERAL BARRIER
}

BY

ZBIGNIEW PALMOWS K I* (WROCŁAW) AND PRZEMYSŁAW Ś W IĄT T K (WROCŁAW)

\author{
Dedicated to Professor Tomasz Rolski
}

\begin{abstract}
In this paper we analyze a Lévy process reflected at a general (possibly random) barrier. For this process we prove the Central Limit Theorem for the first passage time. We also give the finite-time first passage probability asymptotics.
\end{abstract}

2010 AMS Mathematics Subject Classification: Primary: 60G51, 60G50; Secondary: 60K25.

Key words and phrases: Reflected Lévy process, Central Limit Theorem, asymptotics, first passage probability.

\section{INTRODUCTION}

In this paper we consider a general Lévy process reflected at a general (possibly random) barrier. In dimensions greater than one the existence of the reflected process (even for Brownian motion in a time-independent domain) is a non-trivial matter. Some smoothness assumptions on the domain are required (see [20]). However, in one dimension there are no such problems (see [14]).

The motivation for considering reflected Lévy process comes mainly from various storage, queueing and telecommunication models (see [2], [18]). Usually one deals with reflecting at the constant zero barrier producing the workload of the Lévy-driven queue (see [7] for survey). There are many applications where more general reflected Lévy processes appear though. One can consider, for example, models where a constant input is added to the netput mechanism. This additional constant input is not available on liquid basis and can be removed only after some

* This work is partially supported by the National Science Centre under the grant DEC2013/09/B/ST1/01778. The first author kindly acknowledges a partial support by the project RARE318984, a Marie Curie IRSES Fellowship within the Seventh European Community Framework Programme. 
(possibly random) maturity date. Therefore, it can be described in the model by some downward barrier (see, e.g., [15]).

There have been many works on overflow probabilities which correspond to the first-passage time probability of the reflected Lévy process. A corresponding interpretation for the Cramér-Lundberg risk model is also possible, where the general barrier represents the future threshold for (continuous) dividend pay out. Another motivation for considering the reflected Lévy process comes from the structural biology which is treated thoroughly in [10]. Finally, considering the reflection of a Lévy process provides an example of a time inhomogeneous process where we can derive quite precise asymptotic results of the first passage probabilities.

In this paper we consider a general reflected Lévy process $X_{t}$ from another, general and independent, Lévy process $Y_{t}$, and a reflected Lévy process $X_{t}$ from the deterministic barrier. We denote this process by $V_{t}$.

The reflection from the independent Lévy processes has been already considered in some particular cases. For example, Burdzy and Nualart [5] were interested in the reflection of a one-dimensional Brownian motion at the path of another Brownian motion (see also Section 3 in [4] for general issues regarding the reflection of one-dimensional Brownian motion). The reflected Lévy process from the deterministic barrier also has already been considered. It was done in the seminal paper of Hansen [IT]. He derived the Cramér asymptotics for the first passage probability, where deterministic, time-dependent barrier tends to $-\infty$ (see also [] and [9]).

We continue this research deriving (in both cases - reflecting from the random process and reflecting from the deterministic barrier) the Central Limit Theorem for the first passage time

$$
\tau(u)=\inf \left\{t \geqslant 0: V_{t}>u\right\} .
$$

We also identify the tail asymptotics of its distribution, where the time horizon goes to infinity depending linearly on the crossing level.

The most intriguing and the main result of this paper is given in Theorem B.3. In this theorem we consider a deterministic, increasing and convex barrier $f$ and a Lévy process $X$ being a compound Poisson process with negative drift:

$$
X_{t}=\sum_{i=1}^{N_{t}} E_{i}-t
$$

Here, all jumps $E_{i}$ have a distribution function $F$ with the asymptotics

$$
\lim _{x \rightarrow \infty} \frac{1-F(x)}{l(x) x^{-\beta} e^{-\alpha x}}=1
$$

for some slowly varying function $l, \beta \in \mathbb{R}$ and $\alpha>0$. The process $N_{t}$ is a Poisson 
process independent of $\left\{E_{i}\right\}$ and with intensity $\lambda$. In this case,

$$
V_{t}=X_{t}-\min \left(0, \inf _{0 \leqslant s \leqslant t}\left(X_{s}-f(s)\right)\right) .
$$

When $X_{t}-f(t) \stackrel{\text { a.s. }}{\rightarrow}-\infty$ as $t \rightarrow \infty$, under some other technical assumptions, we find the following logarithmic asymptotics:

$$
\lim _{u \rightarrow \infty} \frac{1}{u} \log \mathbb{P}\left(\tau(u) \leqslant f^{-1}(c u)\right)=-\alpha(1-c),
$$

where $f^{-1}$ is a right inverse of the lower barrier $f$ and $0<c<1$. The proof of the above statement is based on identifying precise asymptotic lower and upper bounds by constructing simpler, "envelope-like" processes of $V_{t}$ and analyzing their first passage times. We use some geometrical observation and the fluctuation theory of Lévy processes in the proof. The proof is surprisingly more complex, and we do not know how to transfer it to more general Lévy processes.

We organize the paper in the following way. Firstly, in Section $\square$, we demonstrate the results concerning the Central Limit Theorem for both cases (reflecting from random and deterministic barriers). Then, in Section [3, we focus on the asymptotics of the first-passage time probabilities and prove the main result of Theorem 3.3 .

\section{CENTRAL LIMIT THEOREM}

2.1. Reflection from the independent Lévy process. On $\left(\Omega, \mathcal{F},\left\{\mathcal{F}_{t}\right\}_{\{t \geqslant 0\}}, \mathbb{P}\right)$ we define two independent of each other general Lévy processes $\left\{X_{t}\right\}_{t \geqslant 0}$ and $\left\{Y_{t}\right\}_{t \geqslant 0}$. The filtration $\mathcal{F}_{t}$ is a natural right-continuous filtration generated by $\left(X_{t}, Y_{t}\right)$, satisfying usual conditions. We assume in this paper that $\mathbb{E} X_{1}=m_{X}>0$ and that $\mathbb{E} Y_{1}=m_{Y}<\infty$. We put $Z_{t}=X_{t}-Y_{t}$. Observe that $Z$ is also a Lévy process. We define the reflected process $X$ from the barrier $Y$ in the following way:

$$
V_{t}=X_{t}-\inf _{0 \leqslant s \leqslant t}\left(X_{s}-Y_{s}\right)=Z_{t}-\inf _{0 \leqslant s \leqslant t}\left(Z_{s}\right)+Y_{t} .
$$

Our main interest in this section is the Central Limit Theorem for the first passage time:

$$
\tau(u)=\inf \left\{t \geqslant 0: V_{t}>u\right\} .
$$

By $\stackrel{\mathcal{D}}{\rightarrow}, \stackrel{\mathcal{P}}{\rightarrow}$ and $\stackrel{\text { a.s. }}{\rightarrow}$ we denote the convergence in distribution, in probability and almost sure, respectively.

THEOREM 2.1. We have:

(i) If $\mathbb{E} Z_{1}>0$, then

$$
\frac{\tau(u)}{u} \stackrel{\mathcal{P}}{\rightarrow} \frac{1}{m_{X}}, \quad \frac{\tau(u)-u / m_{X}}{\sqrt{u}} \stackrel{\mathcal{D}}{\rightarrow} N\left(0, \frac{\omega_{X}^{2}}{m_{X}^{3}}\right)
$$


as $u \rightarrow \infty$, where $\omega_{Y}^{2}=\operatorname{Var}\left(Y_{1}\right)$ and $N\left(m \sigma^{2}\right)$ denotes a Gaussian random variable with mean $m$ and variance $\sigma^{2}$.

(ii) If $\mathbb{E} Z_{1}<0$, then

$$
\frac{\tau(u)}{u} \stackrel{\mathcal{P}}{\rightarrow} \frac{1}{m_{Y}}, \quad \frac{\tau(u)-u / m_{Y}}{\sqrt{u}} \stackrel{\mathcal{D}}{\rightarrow} N\left(0, \frac{\omega_{Y}^{2}}{m_{Y}^{3}}\right)
$$

as $u \rightarrow \infty$.

In the proof we will need the following lemma.

LEMMA 2.1. (i) If $\mathbb{E} Z_{1}>0$, then

$$
\frac{V_{t}-m_{X} t}{\sqrt{t}} \stackrel{\mathcal{D}}{\rightarrow} N\left(0, \omega_{X}^{2}\right)
$$

as $t \rightarrow \infty$.

(ii) If $\mathbb{E} Z_{1}<0$, then

$$
\frac{V_{t}-m_{Y} t}{\sqrt{t}} \stackrel{\mathcal{D}}{\rightarrow} N\left(0, \omega_{Y}^{2}\right)
$$

as $t \rightarrow \infty$.

Proof. If $\mathbb{E} Z_{1}>0$, then $\inf _{0 \leqslant s \leqslant t} Z_{s} \stackrel{\mathcal{D}}{\rightarrow} Z$ as $t \rightarrow \infty$ for some finite random variable $Z$. Hence

$$
\begin{aligned}
\frac{V_{t}-m_{X} t}{\sqrt{t}} & =\frac{X_{t}-\inf _{0 \leqslant s \leqslant t} Z_{s}-m_{X} t}{\sqrt{t}} \\
& =\frac{X_{t}-m_{X} t}{\sqrt{t}}-\frac{\inf _{0 \leqslant s \leqslant t} Z_{s}}{\sqrt{t}} \stackrel{\mathcal{D}}{\rightarrow} N\left(0, \omega_{X}^{2}\right) .
\end{aligned}
$$

On the other hand, if $\mathbb{E} Z_{1}<0$, then $Z_{t}-\inf _{0 \leqslant s \leqslant t} Z_{s} \stackrel{\mathcal{D}}{\rightarrow} Z^{\prime}$ as $t \rightarrow \infty$ for some finite random variable $Z^{\prime}$. Thus

$$
\begin{aligned}
\frac{V_{t}-m_{Y} t}{\sqrt{t}} & =\frac{Z_{t}-\inf _{0 \leqslant s \leqslant t}\left(Z_{s}\right)+Y_{t}-m_{Y} t}{\sqrt{t}} \\
& =\frac{Z_{t}-\inf _{0 \leqslant s \leqslant t} Z_{s}}{\sqrt{t}}+\frac{Y_{t}-m_{Y} t}{\sqrt{t}} \stackrel{\mathcal{D}}{\rightarrow} N\left(0, \omega_{Y}^{2}\right)
\end{aligned}
$$

as $t \rightarrow \infty$.

Proof of The or em $\underset{2.1}{ }$. Let $\mathbb{E} Z_{1}>0$. Recall that $\inf _{0 \leqslant s \leqslant t} Z_{s} \stackrel{\mathcal{D}}{\rightarrow} Z$ as $t \rightarrow \infty$ for some finite random variable $Z$. From the Law of Large Numbers we have

$$
m_{X}=\lim _{t \rightarrow \infty} \frac{X_{t}}{t}=\lim _{t \rightarrow \infty} \frac{V_{t}+\inf _{0 \leqslant s \leqslant t} Z_{s}}{t}=\lim _{u \rightarrow \infty} \frac{V_{\tau(u)}}{\tau(u)}=\lim _{u \rightarrow \infty} \frac{u}{\tau(u)} .
$$


Now from Lemma 2.1] it follows that

$$
\frac{V_{t}-m_{X} t}{\sqrt{t}} \stackrel{\mathcal{D}}{\rightarrow} N\left(0, \omega_{X}^{2}\right),
$$

and from Anscombe's theorem (see, e.g., [6], Theorem 7.3.2) we get

$$
\frac{V_{\tau(u)}-m_{X} \tau(u)}{\sqrt{\tau(u)}} \stackrel{\mathcal{D}}{\rightarrow} N\left(0, \omega_{X}^{2}\right) \quad \text { as } u \rightarrow \infty .
$$

Thus

$$
\frac{\sqrt{u}}{\sqrt{\tau(u)}} \frac{u-m_{X} \tau(u)}{\sqrt{u}} \stackrel{\mathcal{D}}{\rightarrow} N\left(0, \omega_{X}^{2}\right),
$$

and finally

$$
\frac{u / m_{X}-\tau(u)}{\sqrt{u}} \stackrel{\mathcal{D}}{\rightarrow} N\left(0, \frac{\omega_{X}^{2}}{m_{X}^{3}}\right) .
$$

The proof of the case (ii) is similar.

2.2. Reflection from the deterministic barrier. Our goal is to get similar results for a Lévy process $X$ reflected at some fixed, deterministic, time-dependent and càdlàg boundary $f(t)$. that is,

By $V_{t}$ we denote the general Lévy process $X_{t}$ reflected at the barrier $f(t)$,

$$
V_{t}=X_{t}-\min \left(0, \inf _{0 \leqslant s \leqslant t}\left(X_{s}-f(s)\right)\right) .
$$

Thus, for $f(0) \geqslant 0$ we have

$$
V_{t}=X_{t}-\inf _{0 \leqslant s \leqslant t}\left(X_{s}-f(s)\right) .
$$

For such a process we can prove the Central Limit Theorem for $\tau(u)$ defined in (2.2).

LEMMA 2.2. (i) If $X_{t}-f(t) \stackrel{\text { a.s. }}{\rightarrow}+\infty$, then

$$
\frac{V_{t}-m_{X} t}{\sqrt{t}} \stackrel{\mathcal{D}}{\rightarrow} N\left(0, \omega_{X}^{2}\right) .
$$

(ii) If $X_{t}-f(t) \stackrel{\text { a.s. }}{\rightarrow}-\infty$, then

$$
V_{t} \sim f(t)
$$

where we write

$$
h \sim g \quad \text { if } \lim _{t \rightarrow \infty} h(t) / g(t)=1 .
$$


The proof of Lemma 2.2 is the same as the proof of Lemma 2.1].

The main result of this section is the following theorem.

THEOREM 2.2. (i) If $X_{t}-f(t) \stackrel{\text { a.s. }}{\rightarrow}+\infty$ as $t \rightarrow \infty$, then

$$
\frac{\tau(u)}{u} \stackrel{\mathcal{P}}{\rightarrow} \frac{1}{m_{X}}, \quad \frac{\tau(u)-u / m_{X}}{\sqrt{u}} \stackrel{\mathcal{D}}{\rightarrow} N\left(0, \frac{\omega_{X}^{2}}{m_{X}^{3}}\right)
$$

as $u \rightarrow \infty$.

(ii) If $X_{t}-f(t) \stackrel{\text { a.s. }}{\rightarrow}-\infty$ as $t \rightarrow \infty$, then

$$
\frac{\tau(u)}{f^{-1}(u)} \stackrel{\mathcal{P}}{\rightarrow} 1
$$

as $u \rightarrow \infty$, where $f^{-1}(u)=\inf \{t \geqslant 0: f(t) \geqslant u\}$.

Let

$$
\xi(u)=X_{\tau(u)}-u .
$$

The proof of the above theorem is based on the following fact.

LEMmA 2.3. If $\mathbb{E} X_{1}>0$ and $X_{t}-f(t) \rightarrow+\infty$ a.s. as $t \rightarrow \infty$, then $\tau(u)$ and $\xi(u)$ are asymptotically independent, i.e., for a bounded and continuous function $h$ on $[0, \infty)$ and a bounded continuous function $g_{u}$ (possibly dependent on $u$ ) on the real line for $u>0$, we have

$$
\mathbb{E}\left(h(\xi(u)) g_{u}\left(\frac{\tau(u)-u / m_{X}}{\sqrt{u}}\right)\right) \sim \mathbb{E} f(\xi(\infty)) \mathbb{E} g_{u}(N),
$$

where $N$ is an $N\left(0, \omega_{X}^{2} / m_{X}^{3}\right)$ random variable.

Proof. The proof of this lemma is the same as the proof of Lemma 5.8 in [2], p. 368. In particular, note that this proof does not change if one allows the dependence of the function $g$ on $u$.

Pro of of The or e m 2.2. The case (i) follows directly from Lemma 2.3 . The case (ii) is a consequence of the observation that $\mathbb{P}\left(\tau \leqslant f^{-1}(u)\right) \rightarrow 1$ as $u \rightarrow \infty$.

REMARK 2.1. If $X_{t}-f(t) \stackrel{\text { a.s. }}{\rightarrow}-\infty$, then $\xi(\infty)$ may not exist. Indeed, if we take $f(t)=[t]^{2}$ (where $[t]$ denotes the integer part of $t$ ) and take $X_{t}$ being a Poisson process with rate $\lambda$, then we have

$$
\lim _{n \rightarrow \infty} \xi\left(n^{2}-\frac{1}{2}\right) \stackrel{\mathcal{P}}{\rightarrow} \frac{1}{2}, \quad \lim _{n \rightarrow \infty} \xi\left(n^{2}+\frac{1}{2}\right) \stackrel{\mathcal{P}}{\rightarrow} A,
$$

where $\mathbb{P}(A=\infty)=e^{-\lambda}$ and $\mathbb{P}\left(A=\frac{1}{2}\right)=1-e^{-\lambda}$. 


\section{FINITE-TIME FIRST PASSAGE PROBABILITIES: ASYMPTOTICS}

3.1. Reflection from the independent Lévy process. We start our considerations, as before, from analyzing the first passage probabilities for the general Lévy process $X$ reflected from another, independent Lévy process $Y$; see (2.11) for the formal definition. In this case we can derive the following result.

THEOREM 3.1. (i) If $\mathbb{E} Z_{1}>0$, then, as $u \rightarrow \infty$, for a general function $g$ we have

$$
\begin{aligned}
\mathbb{P}(\tau(u) \leqslant g(u)) & =\mathbb{P}\left(\frac{\tau(u)-u / m_{X}}{\sqrt{u \cdot \omega_{X}^{2} / m_{X}^{3}}} \leqslant \frac{g(u)-u / m_{X}}{\sqrt{u \cdot \omega_{X}^{2} / m_{X}^{3}}}\right) \\
& \sim \mathbb{P}\left(N(0,1) \leqslant \frac{g(u)-u / m_{X}}{\sqrt{u \cdot \omega_{X}^{2} / m_{X}^{3}}}\right),
\end{aligned}
$$

where the symbol $\sim$ is defined in (2.4), and $N(0,1)$ is a standard Gaussian random variable.

(ii) Similarly, if $\mathbb{E} Z_{1}<0$ and $u \rightarrow \infty$, then

$$
\begin{aligned}
\mathbb{P}(\tau(u) \leqslant g(u)) & =\mathbb{P}\left(\frac{\tau(u)-u / m_{Y}}{\sqrt{u \cdot \omega_{Y}^{2} / m_{Y}^{3}}} \leqslant \frac{g(u)-u / m_{Y}}{\sqrt{u \cdot \omega_{Y}^{2} / m_{Y}^{3}}}\right) \\
& \sim \mathbb{P}\left(N(0,1) \leqslant \frac{g(u)-u / m_{Y}}{\sqrt{u \cdot \omega_{Y}^{2} / m_{Y}^{3}}}\right) .
\end{aligned}
$$

Pro of. The assertion of the theorem is a consequence of Theorem 2.].

3.2. Reflection from the deterministic barrier $f$. By Theorem 2.2 , similar results could be derived for the deterministic boundary.

THEOREM 3.2. Assume that

$$
X_{t}-f(t) \stackrel{\text { a.s. }}{\rightarrow} \infty
$$

and that $g$ is a function such that $g(u) \leqslant f^{-1}(u)$. Then we have

$$
\begin{aligned}
\mathbb{P}(\tau(u) \leqslant g(u)) & =\mathbb{P}\left(\frac{\tau(u)-u / m_{X}}{\sqrt{u \cdot \omega_{X}^{2} / m_{X}^{3}}} \leqslant \frac{g(u)-u / m_{X}}{\sqrt{u \cdot \omega_{X}^{2} / m_{X}^{3}}}\right) \\
& \sim N\left(\frac{g(u)-u / m_{X}}{\sqrt{u \cdot \omega_{X}^{2} / m_{X}^{3}}}\right) .
\end{aligned}
$$


From now on we assume that the following complementary condition holds:

$$
X_{t}-f(t) \stackrel{\text { a.s. }}{\rightarrow}-\infty .
$$

Moreover, we assume that

$$
f(t) \text { is an increasing and convex function, }
$$

and that $X$ is a compound Poisson process given by

$$
X_{t}=\sum_{i=1}^{N_{t}} E_{i}-t,
$$

where generic $E$ has a distribution function $F$, and $N_{t}$ is a Poisson process independent of $\left\{E_{i}, i \in \mathbf{N}\right\}$ and with intensity $\lambda$. Additionally, we will assume that

$$
\bar{F}(x)=1-F(x) \sim l(x) x^{-\beta} e^{-\alpha x}
$$

for some slowly varying function $l$ and $\beta \in \mathbb{R}, \alpha>0$.

From [3] we know that the slowly varying function $l$ has the representation

$$
l(x)=\gamma(x) \exp \left(\int_{a}^{x} \frac{h(u)}{u} d u\right), \quad x \geqslant a
$$

for some $a>0$, where $\gamma(\cdot)$ is measurable and $\gamma(x) \rightarrow \gamma \in(0, \infty), h(x) \rightarrow 0$ as $x \rightarrow 0$.

We also assume that

$$
x \gamma^{\prime}(x) \rightarrow 0 \quad \text { as } x \rightarrow \infty .
$$

REMARK 3.1. Note that, for example, when we take $\gamma(x)=1, a=e$ and $h(x)=1 / \log (x)$, we will get $l(x)=\log (x)$.

This case is the most difficult one since the shape of the barrier has the influence on the behaviour of the reflected process (compare, e.g., with [11] where (B.D) is assumed). Therefore, we manage to derive the asymptotics only under the above restricted assumptions. We believe though that at least the logarithmic asymptotic holds true for a more general setup.

The next theorem is the main result of this paper.

THEOREM 3.3. Assume that (3.2)-(3.7) hold and $\beta>3$. Then, for any $c \in$ $(0,1)$, we have

$$
\lim _{u \rightarrow \infty} \frac{1}{u} \log \mathbb{P}\left(\tau(u) \leqslant f^{-1}(c u)\right)=-\alpha(1-c) .
$$

REMARK 3.2. In fact, in the proof we derive more exact lower and upper uniform bounds, which seems to be of own interest. 
We divide the proof of the theorem into two parts, proving lower and upper bounds for $\mathbb{P}\left(\tau(u) \leqslant f^{-1}(c u)\right)$, both of which hold without the assumption of $\beta>3$.

\section{Lower bound.}

Proposition 3.1. We have

$$
\lim _{u \rightarrow \infty} \frac{\mathbb{P}\left(\tau(u) \leqslant f^{-1}(c u)\right)}{\lambda e^{-\alpha(1-c) u} \frac{l(u-c u)}{\alpha f^{\prime}\left(f^{-1}(c u)\right)}(u-c u)^{-\beta}} \geqslant 1 .
$$

Pr o o f. To get the lower bound of $\mathbb{P}\left(\tau(u) \leqslant f^{-1}(c u)\right)$, we consider the process

$$
\underline{V}_{t}=f(t)+\Delta V_{t},
$$

where $\Delta V_{t}$ is the size of the jump of $V_{t}$ that occurs exactly at time $t>0$. Observe that $V_{t} \geqslant \underline{V}_{t}$. Indeed, note that $V_{t}-V_{t-}+\Delta V_{t} \geqslant f(t)$ and $V_{t-} \geqslant f(t)$ by the definition of the reflected proces $V$. Let us put $\underline{\tau}(u)=\inf \left\{t \geqslant 0: \underline{V}_{t}>u\right\}$. It is clear that

$$
\mathbb{P}\left(\tau(u) \leqslant f^{-1}(c u)\right) \geqslant \mathbb{P}\left(\underline{\tau}(u) \leqslant f^{-1}(c u)\right) .
$$

We also introduce events $A_{k}$ such that there are exactly $k$ jumps of $\underline{V}_{t}$ (and also $V_{t}$ ) before time $f^{-1}(c u)$. Then

$$
\mathbb{P}\left(\underline{\tau}(u) \leqslant f^{-1}(c u)\right)=\sum_{k=1}^{\infty} \mathbb{P}\left(\underline{\tau}(u) \leqslant f^{-1}(c u) \mid A_{k}\right) \mathbb{P}\left(A_{k}\right),
$$

where $\mathbb{P}\left(A_{k}\right)=\frac{\left(\lambda f^{-1}(c u)\right)^{k}}{k !} e^{-\lambda f^{-1}(c u)}$. Besides that conditioned on $A_{k}$ the times of jumps have the same law as the order statistics of $k$ independent uniform distributed random variables on the interval $\left[0, f^{-1}(c u)\right]$. Thus

$$
\mathbb{P}\left(\underline{\tau}(u) \leqslant f^{-1}(c u) \mid A_{k}\right)=1-\left[1-\frac{1}{f^{-1}(c u)} \int_{0}^{f^{-1}(c u)} \bar{F}(u-f(x)) d x\right]^{k}
$$

and

$$
\begin{aligned}
& \mathbb{P}\left(\underline{\tau}(u) \leqslant f^{-1}(c u)\right) \\
= & \sum_{k=1}^{\infty} \frac{\left(\lambda f^{-1}(c u)\right)^{k}}{k !} e^{-\lambda f^{-1}(c u)}\left[1-\left[1-\frac{1}{f^{-1}(c u)} \int_{0}^{f^{-1}(c u)} \bar{F}(u-f(x)) d x\right]^{k}\right] \\
= & 1-\exp \left(-\lambda \int_{0}^{f^{-1}(c u)} \bar{F}(u-f(x)) d x\right) .
\end{aligned}
$$


Hence

$$
\mathbb{P}\left(\underline{\tau}(u) \leqslant f^{-1}(c u)\right) \sim \lambda \int_{0}^{f^{-1}(c u)} \bar{F}(u-f(x)) d x .
$$

To identify more precisely the above asymptotics, we need to analyze asymptotic behaviour of the expression

$$
\lambda \int_{0}^{f^{-1}(c u)} l(u-f(x))(u-f(x))^{-\beta} e^{-\alpha(u-f(x))} d x .
$$

Using the change of variables $f(x)=z$, we get

$$
\begin{aligned}
\int_{0}^{f^{-1}(c u)} l(u-f(x))(u & -f(x))^{-\beta} e^{-\alpha(u-f(x))} d x \\
& =e^{-\alpha u} \int_{f(0)}^{c u} l(u-z)(u-z)^{-\beta} e^{\alpha z} \frac{1}{f^{\prime}\left(f^{-1}(z)\right)} d z
\end{aligned}
$$

We define

$$
g(u, z):=l(u-z)(u-z)^{-\beta} e^{\alpha z} \frac{1}{f^{\prime}\left(f^{-1}(z)\right)}
$$

and put

$$
I(u)=\int_{f(0)}^{c u} g(u, z) d z, \quad J(u)=\frac{g(u, c u)}{\alpha} .
$$

Now, using de l'Hospital's rule we will show that

$$
\frac{I(u)}{J(u)} \rightarrow 1 \quad \text { as } u \rightarrow \infty
$$

Indeed, note that

$$
\begin{aligned}
\frac{d I(u)}{d u}= & l(u-c u)(u-c u)^{-\beta} e^{\alpha c u} \frac{c}{f^{\prime}\left(f^{-1}(c u)\right)} \\
& -\beta \int_{f(0)}^{c u} l(u-z)(u-z)^{-\beta-1} e^{\alpha z} \frac{1}{f^{\prime}\left(f^{-1}(z)\right)} d z \\
& +\int_{f(0)}^{c u} \frac{\partial l(u-z)}{\partial u}(u-z)^{-\beta} e^{\alpha z} \frac{1}{f^{\prime}\left(f^{-1}(z)\right)} d z .
\end{aligned}
$$

Moreover, we have

$$
\frac{\partial l(u-z)}{\partial u}=l(u-z) \frac{\partial \gamma(u-z)}{\partial u} \frac{1}{\gamma(u-z)}-l(u-z) \frac{h(u-z)}{u-z} .
$$


Hence we can write

$$
\begin{aligned}
\frac{d I(u)}{d u}= & c g(u, c u)-\int_{f(0)}^{c u} g(u, z) \frac{\beta}{u-z} d z \\
& +\int_{f(0)}^{c u} g(u, z) \frac{1}{\gamma(u-z)} \frac{\partial \gamma(u-z)}{\partial u} d z-\int_{f(0)}^{c u} g(u, z) \frac{h(u-z)}{u-z} d z .
\end{aligned}
$$

The last two terms are asymptotically negligible because we assumed in (B.6) and (B.7) that both $x \gamma^{\prime}(x) \rightarrow 0$ and $h(x) \rightarrow 0$ as $x \rightarrow \infty$. Note also that the first increment dominates the second one. Furthermore, we can observe that

$$
\begin{aligned}
\frac{d J(u)}{d u}= & -\frac{\beta(1-c)}{\alpha(u-c u)} g(u, c u)+c g(u, c u)-g(u, c u) \frac{c f^{\prime \prime}\left(f^{-1}(c u)\right)}{\alpha^{2} f^{\prime}\left(f^{-1}(c u)\right)^{2}} \\
& +\frac{\gamma^{\prime}(u-c u)(1-c)}{\alpha \gamma(u-c u)} g(u, c u)-(1-c) \frac{h(u-c u)}{\alpha(u-c u)} g(u, c u)
\end{aligned}
$$

is asymptotically equivalent to $c g(u, c u)$ when $f^{\prime \prime}\left(f^{-1}(c u)\right) / f^{\prime}\left(f^{-1}(c u)\right)^{2} \rightarrow 0$ as $u \rightarrow \infty$. We can rewrite this condition as follows:

$$
\frac{f^{\prime \prime}(z)}{f^{\prime}(z)^{2}}=\left[-\frac{1}{f^{\prime}(z)}\right]^{\prime} \rightarrow 0 \quad \text { as } z \rightarrow \infty .
$$

It is satisfied for any increasing convex function $f(z)$ since $-1 / f^{\prime}(z)$ is a negative increasing function bounded by zero. Thus, it must have a limit as $z \rightarrow \infty$. This implies that the derivative of this function vanishes at infinity.

We proved thus that both $d I(u) / d u$ and $d J(u) / d u$ are asymptotically equivalent to $c g(u, c u)$ as $u \rightarrow \infty$. We can now conclude that

$$
\begin{aligned}
\mathbb{P}\left(\underline{\tau}(u) \leqslant f^{-1}(c u)\right) & \sim \lambda e^{-\alpha u} I(u) \sim \lambda e^{-\alpha u} J(u) \\
& =\lambda l(u-c u)(u-c u)^{-\beta} e^{-\alpha((1-c) u)} \frac{1}{\alpha f^{\prime}\left(f^{-1}(c u)\right)},
\end{aligned}
$$

which completes the proof.

\section{Upper bound.}

Proposition 3.2. For any $0<\epsilon<\alpha$ there exist $\theta^{\epsilon}$ and a constant $C_{0}$ such that

$\mathbb{P}\left(\tau(u) \leqslant f^{-1}(c u)\right) \leqslant \frac{C_{0}}{(1+u-c u)^{2}} \exp \left(-(\alpha-\epsilon)\left(u-c u-f^{-1}(c u)\right)-\theta^{\epsilon} f^{-1}(c u)\right)$. 
Pr o o f. To get the upper bound, we consider the following process:

$$
\bar{V}_{t}=c u+f^{-1}(c u)+X_{t} .
$$

Note that $\bar{V}$ will never reach the barrier $f$ before time $f^{-1}(c u)$ because it starts at the level $c u+f^{-1}(c u)$. This observation leads to the conclusion that

$$
\begin{aligned}
\mathbb{P}\left(\tau(u) \leqslant f^{-1}(c u)\right) & \leqslant \mathbb{P}\left(\max _{0 \leqslant s \leqslant f^{-1}(c u)} \bar{V}_{s} \geqslant u\right) \\
& =\mathbb{P}\left(\max _{0 \leqslant s \leqslant f^{-1}(c u)} X_{s} \geqslant u-c u-f^{-1}(c u)\right) \\
& =\mathbb{P}\left(\bar{\tau}\left(u-c u-f^{-1}(c u)\right) \leqslant f^{-1}(c u)\right),
\end{aligned}
$$

where

$$
\bar{\tau}(x)=\inf \left\{t \geqslant 0: X_{t}>x\right\} .
$$

To estimate the above probability, we will use asymptotics of the renewal function given in [13]. We denote by $L$ a local time related to the running supremum of $X$, and by $L^{-1}$ its right-continuous inverse. Finally, let $H_{t}=X_{L_{t}^{-1}}$. The process $\left\{\left(L_{t}^{-1}, H_{t}\right), t \geqslant 0\right\}$ is an ascending ladder height process. We introduce also the sequence $\left\{e_{i}\right\}_{i \in \mathbf{N}}$ of independent exponentially distributed random variables with intensity $q$. Let $\sigma_{n}=\sum_{i=1}^{n} e_{i}$. Now we consider a two-dimensional random walk

$$
\left\{\left(S_{i}, R_{i}\right)=\left(L_{\sigma_{i}}^{-1}, H_{\sigma_{i}}\right), i=1,2, \ldots\right\}
$$

starting from $(0,0)$ and having the step size distribution

$$
\mu(d x, d t)=\mathbb{P}\left(H_{\sigma_{1}} \in d x, L_{\sigma_{1}}^{-1} \in d t\right) .
$$

Let us put

$$
\Upsilon(x)=\min \left\{n: S_{n}>x\right\}, \quad G(x, t)=\mathbb{P}\left(\Upsilon(x)<\infty, R_{\Upsilon(x)} \leqslant t\right) .
$$

Then from [19] we have

$$
\mathbb{P}(\bar{\tau}(x) \leqslant t) \leqslant \frac{G(x, t+M)}{h(0-, M)}
$$

for any $M, x, t>0$, where

$$
G(x, t)=\sum_{n=0}^{\infty} \mu^{\star n} \star h(x, t)
$$

is a renewal function, and $h(x, t):=\mathbb{P}\left(H_{\sigma_{1}}>x, L_{\sigma_{1}} \leqslant t\right)$ with $h(0-, M)=$ $\lim _{x \uparrow 0} h(x, M)$. 
Let us denote by $\Pi_{H}$ the Lévy measure associated with the ladder height process $H$. Then, by Vigon's formula (see, e.g., [18], Corollary 7.9) for the spectrally positive Lévy process $X$, we have

$$
\bar{\Pi}_{H}(y)=\int_{0}^{\infty} e^{-\Phi(0) z} \bar{\Pi}_{X}(z+y) d z
$$

where

$$
\Phi(\theta)=\inf \left\{\kappa \geqslant 0: \log \mathbb{E} e^{-\kappa X_{1}} \geqslant \theta\right\}
$$

is the right inverse of the Laplace exponent of the dual process $-X$. Hence

$$
\frac{\bar{\Pi}_{H}(y)}{\bar{\Pi}_{X}(y)}=\int_{0}^{\infty} e^{-\Phi(0) z} \frac{\bar{\Pi}_{X}(z+y) d z}{\bar{\Pi}_{X}(y)} \sim \int_{0}^{\infty} e^{-\Phi(0) z} e^{-\alpha z} d z=\frac{1}{\alpha+\Phi(0)} .
$$

Moreover, by Lemma 3.5 in [17] we obtain

$$
\mathbb{P}\left(H_{\sigma_{1}}>x\right)=q \int_{0}^{\infty} e^{-q t} \mathbb{P}\left(H_{t}>x\right) d t \sim q \int_{0}^{\infty} e^{-q t} t\left(\mathbb{E} e^{\alpha H_{1}}\right)^{t-1} \bar{\Pi}_{H}(x) d t .
$$

From (B.12) we can conclude now that

$$
\mathbb{P}\left(H_{\sigma_{1}}>x\right)=C_{1} \bar{\Pi}_{H}(x) \sim C_{2} \bar{\Pi}_{X}(x) \sim C_{2} \lambda l(x) x^{-\beta} e^{-\alpha x},
$$

where $C_{1}=q \int_{0}^{\infty} e^{-q t} t\left(E e^{\alpha H_{1}}\right)^{t-1} d t$ and $C_{2}=C_{1} /(\alpha+\Phi(0))$. Let us put

$$
\Theta=\left\{\left(\theta_{1}, \theta_{2}\right) \in \mathbb{R}^{2}: \int|(x, t)| \exp \left(\theta_{1} x+\theta_{2} t\right) \mu(d x, d t)<\infty\right\} .
$$

From the equation (B.13) it follows that for any $0<\epsilon<\alpha$ there exists $\theta^{\epsilon}$ such that $\left(\alpha-\epsilon, \theta^{\epsilon}\right) \in \Theta$ and

$$
\mathbb{E} \exp \left((\alpha-\epsilon) H_{\sigma_{1}}+\theta^{\epsilon} L_{\sigma_{1}}^{-1}\right)=1 .
$$

Moreover, we have also

$$
\mathbb{E}\left[\left(H_{\sigma_{1}}+L_{\sigma_{1}}^{-1}\right)^{2} \exp \left((\alpha-\epsilon) H_{\sigma_{1}}+\theta^{\epsilon} L_{\sigma_{1}}^{-1}\right)\right]<\infty .
$$

Hence, from Theorem 1.2 of [1]3] applied with $\left(c_{1}, c_{2}\right)=(1,1)$ we obtain

$$
G(x, t+M) \leqslant C_{0}(1+|x+(t+M)|)^{-2} \exp \left(-(\alpha-\epsilon) x-\theta_{2}^{\epsilon}(t+M)\right) .
$$

Putting $x=u-c u-f^{-1}(c u)$ and $t=f^{-1}(c u)$ into the equation (B.II) and applying it to (3.10) and (3.14), we get

$$
\begin{aligned}
& \mathbb{P}\left(\bar{\tau}\left(u-c u-f^{-1}(c u)\right) \leqslant f^{-1}(c u)\right) \\
& \quad \leqslant \frac{C_{0}\left(1+\left|\left(u-c u-f^{-1}(c u)\right)+\left(f^{-1}(c u)+M\right)\right|\right)^{-2}}{h(0-, M)} \\
& \quad \times \exp \left(-(\alpha-\epsilon)\left(u-c u-f^{-1}(c u)\right)-\theta_{2}^{\epsilon}\left(f^{-1}(c u)+M\right)\right) .
\end{aligned}
$$


Sending $q \rightarrow \infty$ (note that then $h(0-, M) \rightarrow 1$ ) and then sending $M \rightarrow 0$ complete the proof.

REMARK 3.3. For $\alpha \geqslant 0$ and $\beta>3$ one can take $\epsilon \downarrow 0$ in the assertion of Proposition 3.2 to get the upper bound independent of $\epsilon$ since then $\theta_{2}^{\epsilon} \rightarrow \theta^{0}$ for some fixed $\theta^{0}$ and constant $C_{0}$ is bounded above.

Pro of of The or e $m$ 3.3. The assertion of the theorem follows from Propositions 3.1 and 3.2 where we take $\epsilon \downarrow 0$ in the latter.

REMARK 3.4. Let $f(t)=t^{2}, \beta>3$ and $\bar{F}(x)=e^{-\alpha x}$. In this case we have

$$
\lim _{u \rightarrow \infty} \frac{1}{u} \log \mathbb{P}(\tau(u) \leqslant \sqrt{c u})=-\alpha(1-c) .
$$

\section{REFERENCES}

[1] J. Abate and W. Whitt, Transient behavior of regulated Brownian motion. I: Starting at the origin, Adv. in Appl. Probab. 19 (3) (1987), pp. 560-598.

[2] S. Asmussen, Applied Probability and Queues, second edition, Springer, New York 2003.

[3] N. H. Bingham, C. M. Goldie, and J. L Teugels, Regular Variation, Cambridge University Press, Cambridge 1989.

[4] K. Burdzy, Z.-Q. Chen, and J. Sylvester, The heat equation and reflected Brownian motion in time-dependent domains, Ann. Probab. 32 (1) (2004), pp. 775-804.

[5] K. Burdzy and D. Nualart, Brownian motion reflected on Brownian motion, Probab. Theory Related Fields 122 (4) (2002), pp. 471-493.

[6] K. L. Chung, A Course in Probability Theory, second edition, Academic Press, New York 1974.

[7] K. Dębicki and M. Mandjes, Lévy driven queues, Surv. Oper. Res. Manag. Sci. 17 (1) (2012), pp. 15-37.

[8] R. A. Doney and R. A. Maller, Cramér's estimate for a reflected Lévy process, Ann. Appl. Probab. 15 (2) (2005), pp. 1445-1450.

[9] N. R. Hansen, The maximum of a random walk reflected at a general barrier, Ann. Appl. Probab. 16 (1) (2006), pp. 15-29.

[10] N. R. Hansen, Asymptotics for local maximal stack scores with general loop penalty function, Adv. in Appl. Probab. 39 (3) (2007), pp. 776-798.

[11] N. R. Hansen, The maximum of a Lévy process reflected at a general barrier, Stochastic Process. Appl. 119 (2009), pp. 2336-2356.

[12] J. M. Harris on, Brownian Motion and Stochastic Flow Systems, Wiley, New York 1985.

[13] T. Höglund, An asymptotic expression for the probability of ruin within finite time, Ann. Probab. 18 (1990), pp. 378-389.

[14] O. Kella, Reflecting thoughts, Statist. Probab. Lett. 76 (2006), pp. 1808-1811.

[15] O. Kella, O. Boxma, and M. Mandjes, A Lévy process reflected at a Poisson age process, J. Appl. Probab. 43 (1) (2006), pp. 221-230.

[16] O. Kella and W. Whitt, Useful martingales for stochastic storage processes with Lévy input, J. Appl. Probab. 29 (2) (1992), pp. 396-403.

[17] C. Klüppelberg, A. E. Kyprianou, and R. A. Maller, Ruin probabilities and overshoots for general Lévy insurance risk processes, Ann. Appl. Probab. 14 (4) (2004), pp. 1766 1801. 
[18] A. E. Kyprianou, Introductory Lectures on Fluctuations of Lévy Processes with Applications, Springer, Berlin 2006.

[19] Z. Palmowski and M. Pistorius, Cramér asymptotics for finite time first passage probabilities of general Lévy processes, Statist. Probab. Lett. 79 (2009), pp. 1752-1758.

[20] H. Tanaka, Stochastic differential equations with reflecting boundary conditions in convex regions, Hiroshima Math. J. 9 (1979), pp. 163-177.

Zbigniew Palmowski

Faculty of Pure and Applied Mathematics

Wrocław University of Science and Technology

Wybrzeże Wyspiańskiego 27

50-370 Wrocław, Poland

E-mail: zbigniew.palmowski@gmail.com
Przemysław Świątek

Credit Suisse

pl. Grunwaldzki 25

50-365 Wrocław, Poland

E-mail:przemek.swiatek@gmail.com

Received on 31.8.2016;

revised version on 21.4.2017 